\title{
Programmatic Access to Logical Models in the Cell Collective Modeling Environment via a REST API
}

\author{
Bryan M Kowal ${ }^{1}$, Travis R Schreier ${ }^{1}$, Joseph T Dauer ${ }^{2}$, Tomáš Helikar $^{1 *}$ \\ 1 Department of Biochemistry, 2 School of Natural Resources, University of Nebraska- \\ Lincoln, Lincoln, NE, USA \\ * Corresponding author: thelikar2@unl.edu
}

\section{Abstract}

Summary: Cell Collective (www.cellcollective.org) is a web-based interactive environment for constructing, simulating and analyzing logical models of biological systems. Herein, we present a Web service to access models, annotations, and simulation data in the Cell Collective platform through the Representational State Transfer (REST) Application Programming Interface (API). The REST API provides a convenient method for obtaining Cell Collective data through almost any programming language. To ensure easy processing of the retrieved data, the request output from the API is available in a standard JSON format.

Availability and Implementation: The Cell Collective REST API is freely available at http://thecellcollective.org/tccapi. All public models in Cell Collective are also available through the REST API. For users interested in creating and accessing their own models through the REST API first need to create an account in Cell Collective (http://thecellcollective.org).

Contact: thelikar2@unl.edu

Supplementary Information: Technical user documentation: https://goo.gl/U52GWo

Keywords: logical models; Boolean models; REST API; systems biology

\section{Introduction}

Cell Collective is an interactive platform for the creation, simulations, and analyses of largescale logical models (http://thecellcollective.org; (Helikar et al., 2015, 2013; Tomas Helikar et al., 2012) $\square$ ). The web-based software has been designed to make computational network modeling more accessible to the modeling, experimental, and life sciences education communities. The platform provides collaborative model creation, editing, and simulation that do not require manual editing of complex mathematical expressions, or writing code to execute these expressions. In addition, the Cell Collective Knowledge Base enables users to fully annotate every model interaction with supporting literature, providing an environment with fully transparent models that can be easily extended by others within a single platform. Currently, Cell Collective contains over 50 published logical models that are available to the community for simulations, analyses, and additional modifications. The platform also contains over 1,500 private models created by Cell Collective users. Models can be created, edited, simulated, and analyzed directly in Cell Collective, or downloaded in multiple formats, 
including truth tables (.csv files), text files with all logical expressions, and the recent SBMLqual format (Chaouiya et al., 2013) $\square$. While other web-based network modeling platforms exist (e.g., Virtual Cell (Slepchenko et al., 2003) $\square$ ), they tend to utilize differential equations for the modeling technique to capture high levels of detail. The Cell Collective platform, on the other hand, utilizes logical models as they are more scalable, and still provide enough dynamical resolution.

Herein, we report a new REST API that provides users with programmatic access to Cell Collective models, simulations, and model annotations. This makes the access to logical models and the biological knowledge supporting them more convenient for analyses by other software tools, such as GINsim (Chaouiya et al., 2012) $\square$, CellNOpt (Terfve et al., 2012) $\square$, BoolNet (Müssel et al., 2010) $\square$, and GNA (Batt et al., 2012) $\square$.

\section{Implementation}

The REST API has been implemented as a Spring Boot Application. The API consists of a number of REST Controllers that have been registered via Spring Web to expose service endpoints that can be accessed over the Internet. The new REST API Controllers utilize the existing Cell Collective pluggable services layer to retrieve information from the Cell Collective database. The REST API has been secured using Spring Security. Service endpoints are available to make the REST API accessible anonymously and with basic HTTP authentication (using Cell Collective credentials).

Every model in Cell Collective is uniquely identified. Associated with each model are simulations and analyses, as well as annotations of each model interaction as entered in the model Knowledge Base. The REST API provides read-only access to all of these components. All API responses are in JSON format. All API responses utilize a standard format to provide a uniform way to distinguish between requests that were successfully handled and requests that the API failed to handle. Detailed documentation for all available functions, and instructions on how to use them are available at: https://goo.gl/U52GWo

\section{Data Privacy}

Cell Collective models are either public or private. Public models are available to anyone for simulations and analyses, and users can make a private copy, that can be edited and/or extended in any way. Private models are available solely to the user who created them. These models can be shared selectively with others within the platform. Users can also create unique, read-only links to the models for anonymous viewings (e.g., to provide reviewers of manuscripts or grants access to the model for review). The API takes into consideration these restrictions through authentication, and provides users with read-only access to all public models. In addition, API users can only access their own private models and simulation results. 


\section{Model Data}

Models in Cell Collective are logical network models, consisting of components and directed interactions between the them. Each component in the model can assume an active or inactive activity level, as governed by the biological regulatory mechanism described with a Boolean function (more details on Boolean networks can be found in various reviews: e.g.,(Albert and Thakar, 2014; Helikar et al., 2010) $\square \square$. To make the models more accessible to the experimental community, each regulatory mechanism is described in biological terms (e.g., positive/negative regulators, conditions/co-factors, sub-conditions, etc.) that are translated into the underlying Boolean function (Tomáš Helikar et al., 2012) $\square$. As such, the API provides access not only to the Boolean functions, but also to the biological description of each component's regulatory mechanism. The mathematical description of a Cell Collective model can be returned by the API as a set of .csv files with each component's truth table, a text file with the individual Boolean functions, as well as an SBML file for the complete model (as described by the SBML extension for qualitative models). The API also provides access to the information in the Cell Collective Knowledge Base (returned as text), containing detailed annotations for each interaction in a model. Finally, the API enables access to model simulations saved in Cell Collective by a user. All available services through the API are summarized in Table 1.

\begin{tabular}{|c|c|}
\hline Service & Description \\
\hline getAvailableModelRef & $\begin{array}{l}\text { Retrieve information }\{\text { id, name, tags }\} \text { for all model(s) that the requester has } \\
\text { access to. Only published models will be returned for non-authenticated } \\
\text { users. All private, shared, and published models will be returned for } \\
\text { authenticated users. }\end{array}$ \\
\hline getModelLogicalExpressions & $\begin{array}{l}\text { Retrieve all logical (Boolean) expressions associated with a model. } \\
\text { Expressions are in the form: X \& !Y |Z with parentheses where required to } \\
\text { ensure proper order of operations. " } \& \text { " represents the AND relationship; "!" } \\
\text { represents negation, and "|" corresponds to the OR relationship. }\end{array}$ \\
\hline getModelSharelnformation & $\begin{array}{l}\text { Retrieve information about collaborators who have shared access to a } \\
\text { model. The response consists of a list of users by username, as well as the } \\
\text { date the model was shared with a given user, and whether or not the user } \\
\text { has accepted the share request. }\end{array}$ \\
\hline exportModel & $\begin{array}{l}\text { Retrieve file bytes for an export of a model in the specified format(s) } \\
\text { \{Expression, Truth Table, SBML\}. The file bytes can be written to a zip file. } \\
\text { The expression export option will include a text file containing all logical } \\
\text { expressions returned by the getModelLogicalExpressions method above. } \\
\text { The truth table export option will return truth tables in separate text files for } \\
\text { each species. Finally, the SBML export option will return the model } \\
\text { converted to SBML Qualitative Models. (Chaouiya et al., 2015, 2013) }\end{array}$ \\
\hline getSpeciesReferences & $\begin{array}{l}\text { Retrieve all model components }\{\text { id, name }\} \text {. The id of the components that } \\
\text { are returned can be utilized within the getModules method to retrieve the } \\
\text { regulatory Modules that are associated with the Component (if any). (See } \\
\text { (Tomas Helikar et al., 2012; Tomáš Helikar et al., 2012) } \square \text { for more } \\
\text { information on how regulatory modules are defined in Cell Collective) }\end{array}$ \\
\hline getModules & $\begin{array}{l}\text { Retrieve all regulatory module(s) associated with a specified model } \\
\text { component. (See (Tomas Helikar et al., 2012; Tomáš Helikar et al., 2012) } \\
\text { for more information on how regulatory modules are defined in Cell }\end{array}$ \\
\hline
\end{tabular}




\begin{tabular}{|c|c|}
\hline & $\begin{array}{l}\text { Collective). The information returned by this method includes the id of the } \\
\text { module, the components that are part of the module, the type of module, } \\
\text { and a description of the regulatory mechanism. The returned module id can } \\
\text { be used to retrieve both dominance information (see below) via the } \\
\text { getDominance API method (when applicable) and to retrieve conditions via } \\
\text { the getConditions API method (when applicable). }\end{array}$ \\
\hline getDominance & $\begin{array}{l}\text { Retrieve all positive regulator module(s) over which a specified negative } \\
\text { module is dominant. (See (Tomáš Helikar et al., 2012) } \square \text { for more details on } \\
\text { dominance between positive and negative regulators). }\end{array}$ \\
\hline getConditions & $\begin{array}{l}\text { Retrieve condition(s) associated with a specified regulatory module. The } \\
\text { information returned by this method includes the condition id, the type of } \\
\text { condition, the name of the condition, the state of the condition, the species } \\
\text { that participate in the condition, and a description of the condition. } \\
\text { The condition id can be also used to retrieve sub-conditions via the } \\
\text { getSubConditions API method (when applicable). (See (Tomáš Helikar et } \\
\text { al., 2012) } \square \text { for more details on conditions and sub-conditions). }\end{array}$ \\
\hline getSubConditions & $\begin{array}{l}\text { Retrieve the sub-condition(s) associated with a specified condition of a } \\
\text { regulatory module. The information returned by this method will include the } \\
\text { sub-condition id, the type of sub-condition, the name of the sub-condition, } \\
\text { the state of the sub-condition, the species that participate in the sub- } \\
\text { condition, and an English description of the sub-condition. See (Tomáš } \\
\text { Helikar et al., 2012) } \square \text { for more details on conditions and sub-conditions). }\end{array}$ \\
\hline getBiologic & $\begin{array}{l}\text { Retrieve all regulatory biologic information (modules, conditions, sub- } \\
\text { conditions, and dominance) for a specified model component. This API } \\
\text { returns a combination of all of the information returned by the: } \\
\text { getSpeciesReferences, getModules, getDominance, getConditions, and } \\
\text { getSubConditions API methods. }\end{array}$ \\
\hline getKBPage & $\begin{array}{l}\text { Retrieve the contents of a Knowledge Base Page for a specified model } \\
\text { component. The information returned by this method includes: the owner of } \\
\text { the Knowledge Base Page, the date/time that the page was last updated, } \\
\text { page content, and any bibliographic information included on the page. }\end{array}$ \\
\hline getDynamicExperiments & $\begin{array}{l}\text { Retrieves saved dynamical analysis experiment(s) for a specified model. } \\
\text { The information returned by this method includes: the id of the experiment, } \\
\text { the name of the experiment, and the settings that were used to run the } \\
\text { experiment (number of simulations, number of time steps, transient time - } \\
\text { see (Helikar and Rogers, 2009) } \square \text { for more details on these parameters). } \\
\text { The experiment id can be used to retrieve the data generated by the } \\
\text { experiment via the getDynamicExperimentData method. }\end{array}$ \\
\hline getDynamicExperimentData & $\begin{array}{l}\text { Retrieves the data generated during the specified experiment. The user can } \\
\text { request either a byte stream of the data that can be used to write in a file on } \\
\text { the REST client or the raw data arranged in a JSON structure. }\end{array}$ \\
\hline searchModels & $\begin{array}{l}\text { Search for model(s) by name or description contents. This method returns } \\
\text { the id and name of the Model(s) that match the search criteria. Note that } \\
\text { only Model(s) that are visible to the user who executed the request are } \\
\text { returned. }\end{array}$ \\
\hline searchSpecies & $\begin{array}{l}\text { Search for model components by name or knowledge base content. This } \\
\text { method returns the id and name of component(s) that match the search } \\
\text { criteria. Note that only Species that belong to Models that are visible to the } \\
\text { user that executed the request are returned. }\end{array}$ \\
\hline
\end{tabular}

Table 1: List of available API services. Note that information can be obtained only for models that are directly available to the user based on proper authentication and authorization. 


\section{Examples}

The following examples illustrate how a user can utilize the API to retrieve a list of available models (Example 1), and how to retrieve information about a specific model (Example 2) in Java and C\#.

Example 1 illustrates how the API can be utilized in Java (Figure 1) and C\# (Figure 2) to acquire and print the id and names of models that are visible to an authenticated user. All models available to a user can be retrieved with the getAvailableModelRef API method (boxed in red in Figure 1). The user also needs to provide a valid username and password to access the models (boxed in blue in Figure 1). Note that the username and password used in this example are placeholders that need to be replaced by real username and password. A sample of the output - IDs and names of models visible to an authenticated user - produced by Example 1 is exemplified in Box 1.

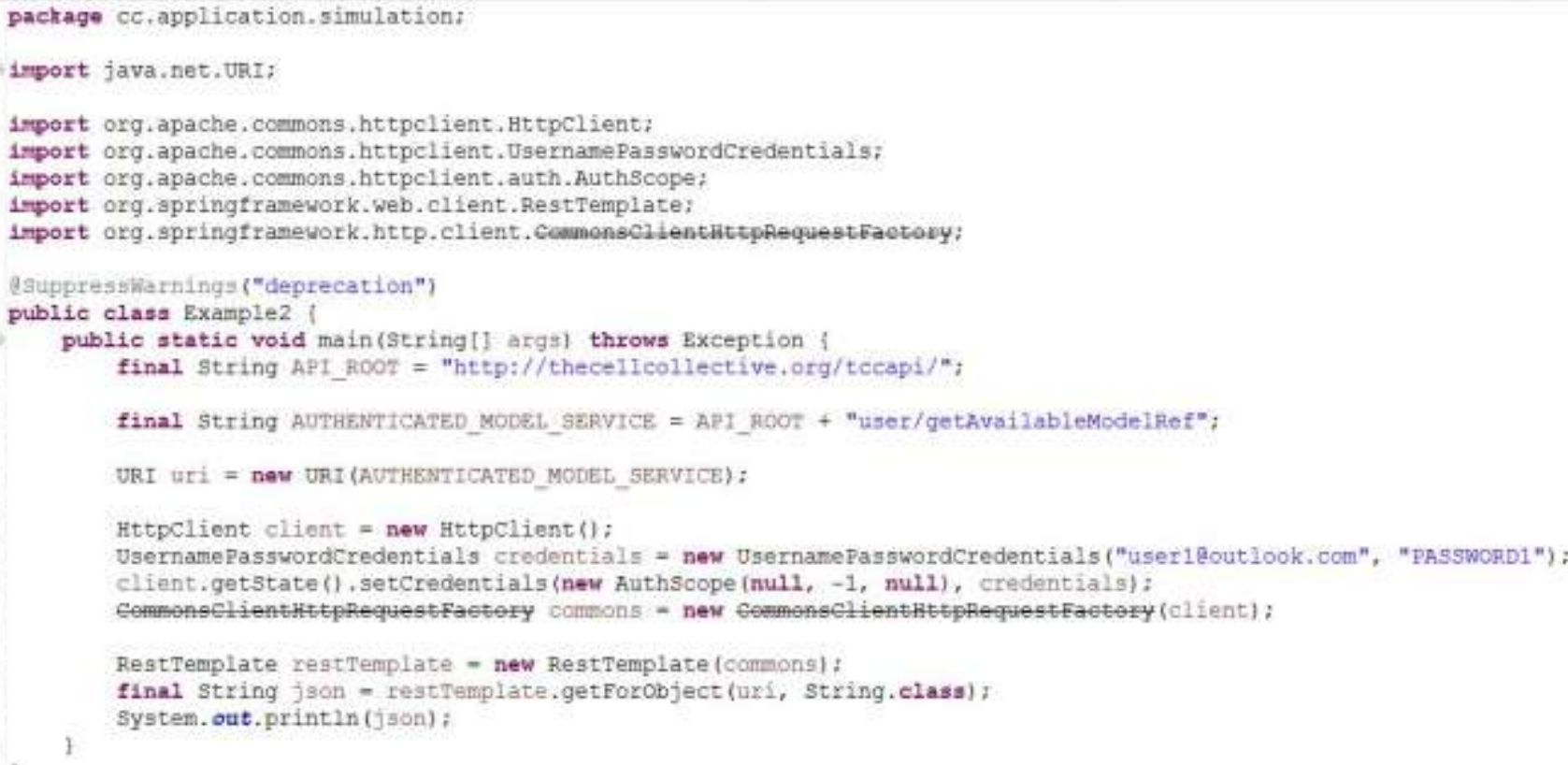

Figure 1: Example (1) utility of the Cell Collective API using Java. An active user account on Cell Collective is required to recreate and/or experiment with this example. No additional parameters are required to access this service. The illustrated code performs the following actions: Declaring the API URLs (Lines 14, 16), Creating a client connection (Line 25), Creating authentication information to supply with the request (Lines 20-23), Retrieving the API response (Line 26), Extracting the JSON response from the API response (Line 26), Printing the JSON that was retrieved to the console (Line 27). 


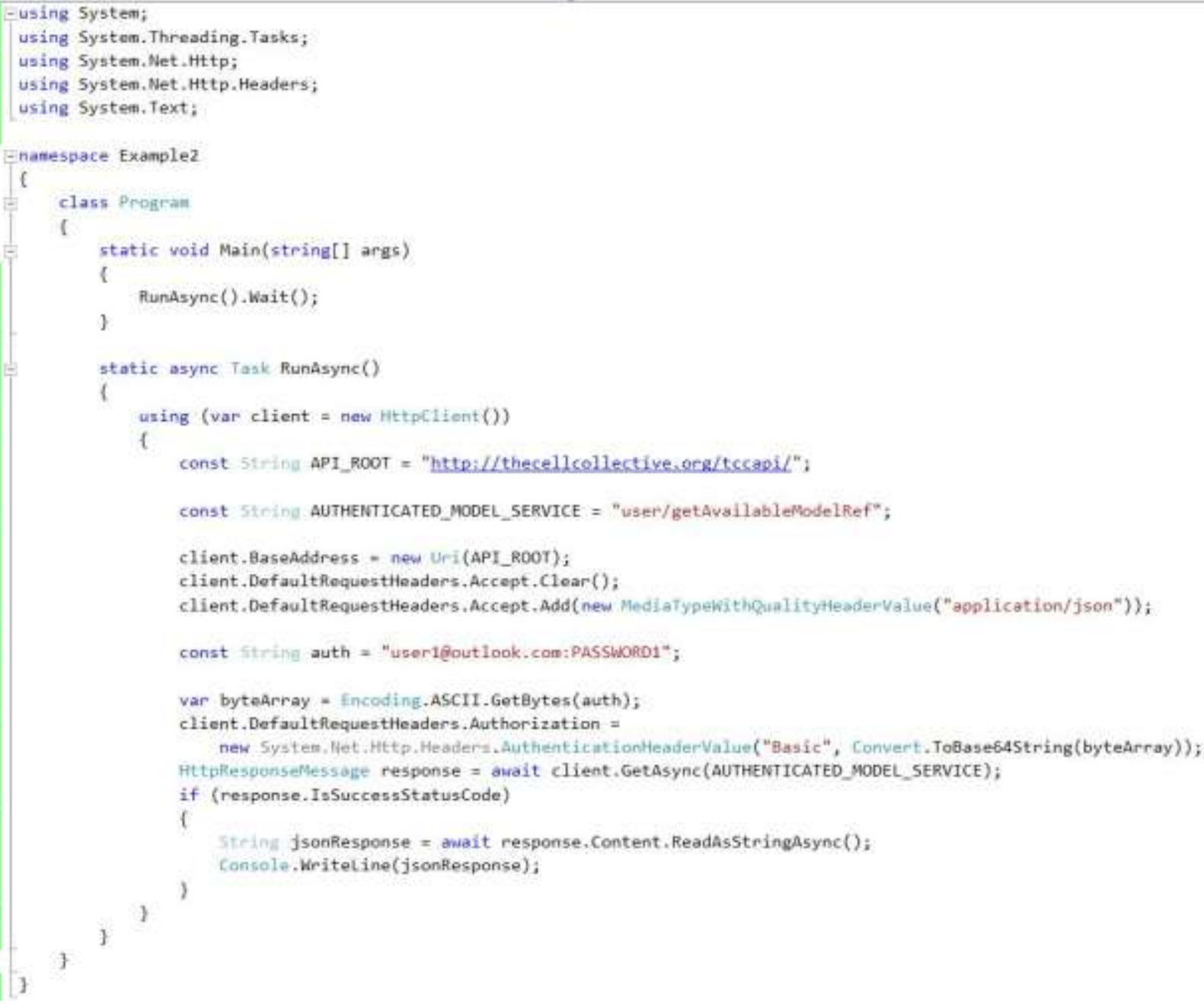

Figure 2. Example (1) utility of the Cell Collective API using C\#. An active user account on Cell Collective is required to recreate and/or experiment with this example. No additional parameters are required to access this service. The illustrated code performs the following actions: Declaring the API URLs (Lines 20, 22), Creating a client connection (Lines 18, $24-$ 26), Creating authentication information to supply with the request (Line 28, $30-32$ ), Retrieving the API response (Line 33), Extracting the JSON response from the API response (Line 36), Printing the JSON that was retrieved to the console (Line 37).

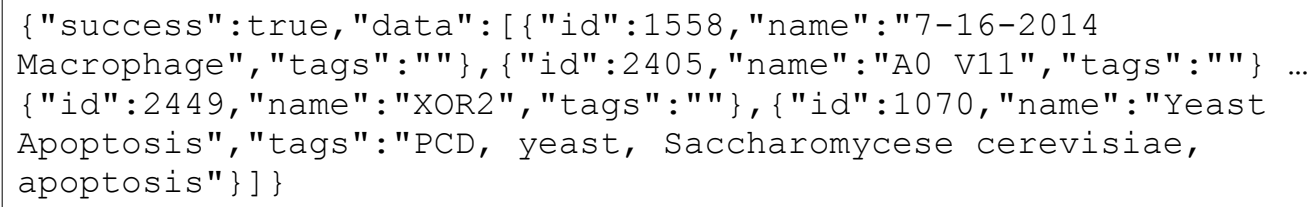

Box 1: A sample JSON response from Example 1, providing a list of models visible to the authenticated user.

Example 2 (Figure 3 and 4) illustrate how the API can be utilized in Java and C\#, respectively, to obtain and print the list (IDs and names) of all components in one of the models in Cell Collective. Required parameters are incorporated directly into the URL. As illustrated above, "8" was a required parameter to indicate which model should be retrieved from the database. 
Parameters are specified as simple "key = value" pairs appended to a URL. A sample of the output - IDs and names of components of the model - produced by Example 2 is exemplified in Box 2.

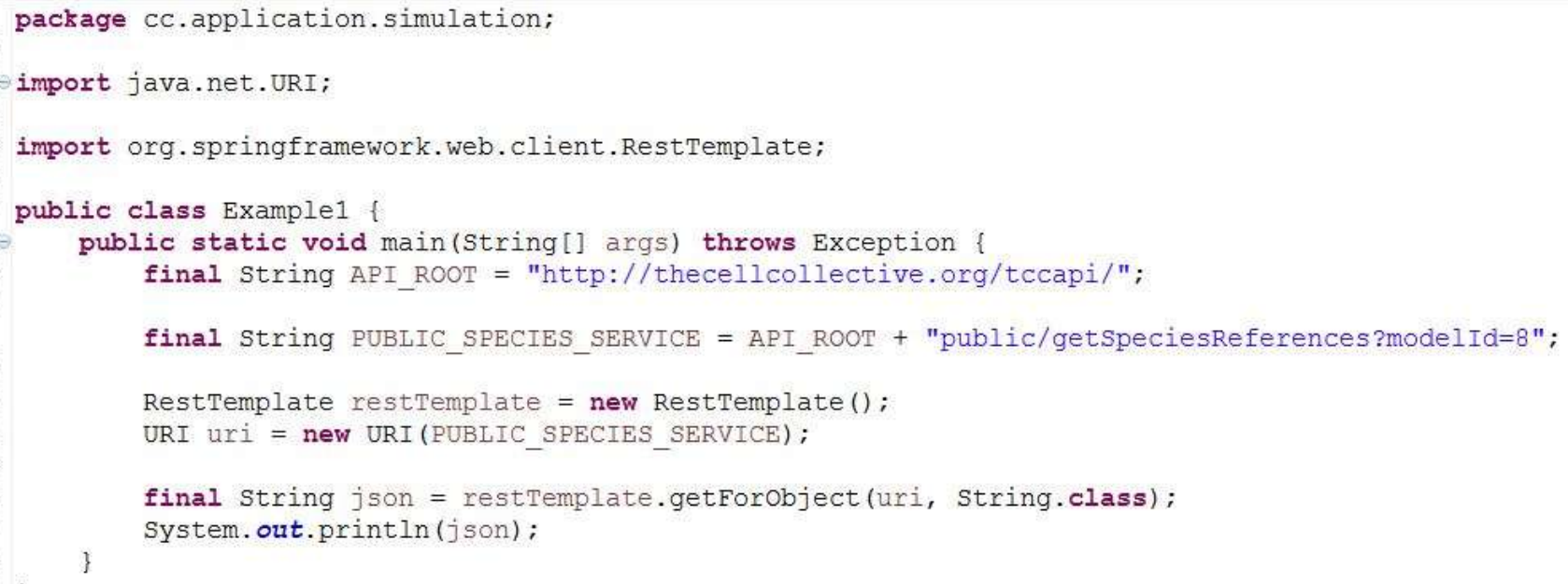

Figure 3: Example (2) utility of the Cell Collective API using Java. The illustrated code performs the following actions: Declaring the API URLs (Lines 9 and 11), Creating a client connection (Lines 13 -14), Retrieving the API response (Line 16 in Java), Extracting the JSON response from the API response (Line 16), Printing the JSON that was retrieved to the console (Line 17). 


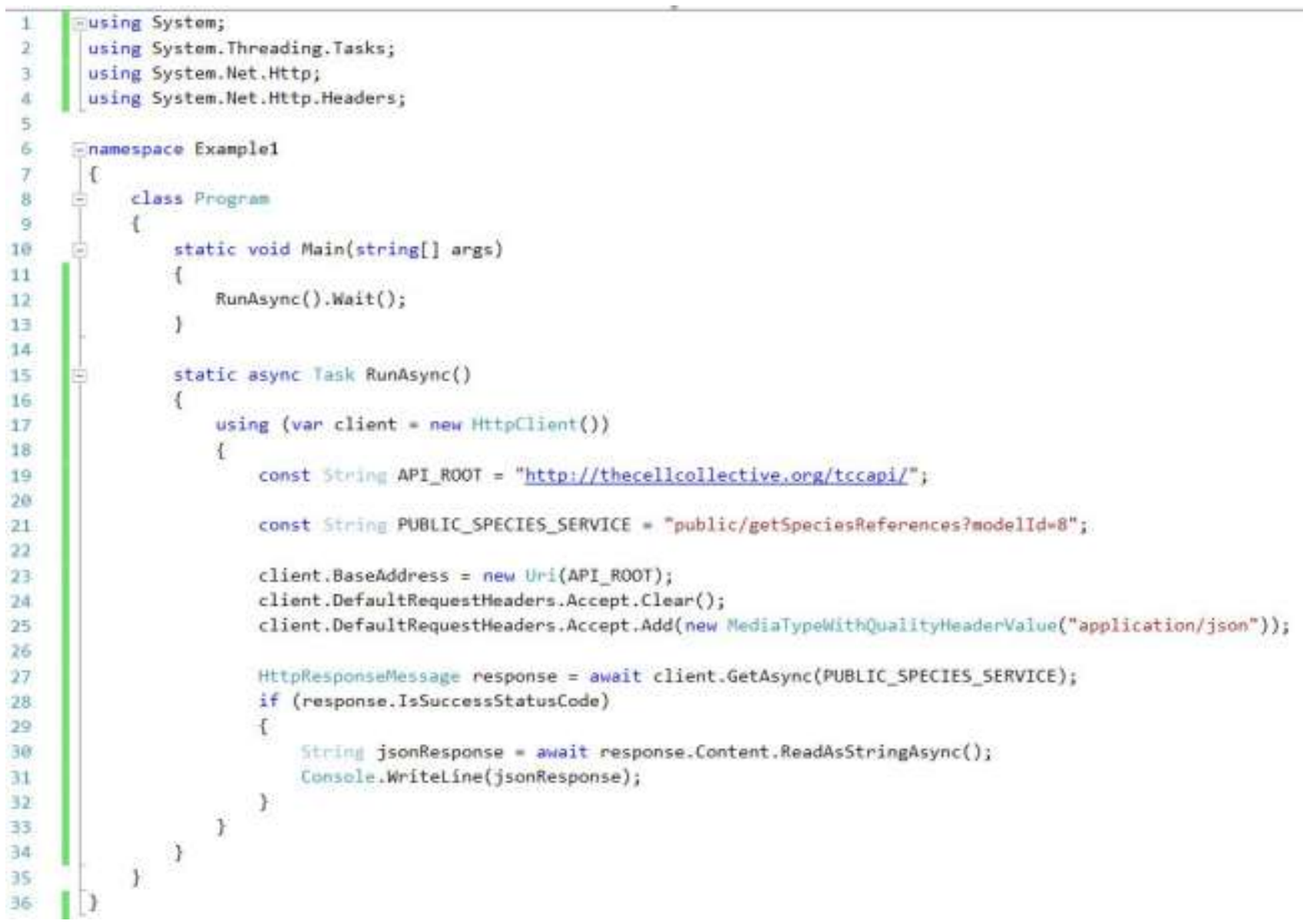

Figure 4: Example (2) utility of the Cell Collective API using C\#. The illustrated code performs the following actions: Declaring the API URLs (Lines 19, 21), Creating a client connection (Lines 17, 23 - 25 ), Retrieving the API response (Line 27), Extracting the JSON response from the API response (Line 30), Printing the JSON that was retrieved to the console (Line 31).

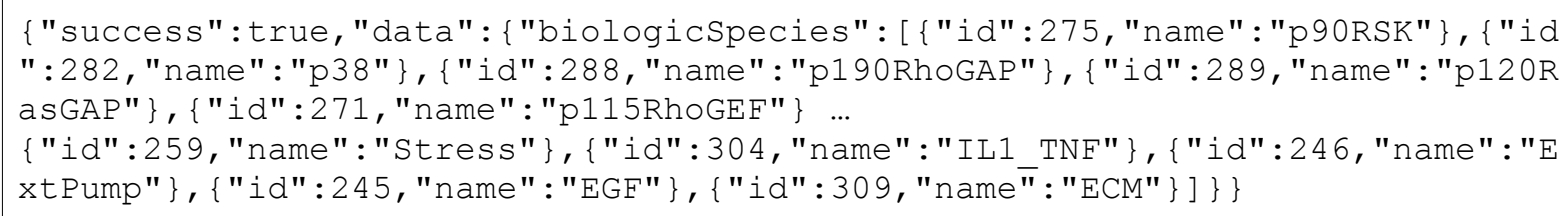

Box 2: A sample JSON response from Example 2, providing the list of components in model \#8 with their associated IDs and names. 


\section{Conclusions}

The Cell Collective REST API can be used to query the models, their annotations, and simulation data from a variety of programming languages. This allows for a flexible programmatic access to logical models, previously only available for download on an individual basis. In the future we plan to expand the API to allow external tools to save data in Cell Collective. This will, for example, enable desktop simulation tools to directly save simulations performed locally on the server, and subsequently make them available to the users via the Cell Collective website.

\section{Acknowledgments}

This work was funded by a grant from the National Science Foundation (\#1432001).

\section{References}

Albert, R., Thakar, J., 2014. Boolean modeling: a logic-based dynamic approach for understanding signaling and regulatory networks and for making useful predictions. Wiley Interdiscip. Rev. Syst. Biol. Med. 6, 353-69.

Batt, G., Besson, B., Ciron, P.-E., de Jong, H., Dumas, E., Geiselmann, J., Monte, R., Monteiro, P.T., Page, M., Rechenmann, F., Ropers, D., 2012. Genetic network analyzer: a tool for the qualitative modeling and simulation of bacterial regulatory networks. Methods Mol. Biol. 804, 439-62. doi:10.1007/978-1-61779-361-5_22

Chaouiya, C., Bérenguier, D., Keating, S.M., Naldi, A., van lersel, M.P., Rodriguez, N., Dräger, A., Büchel, F., Cokelaer, T., Kowal, B., Wicks, B., Gonçalves, E., Dorier, J., Page, M., Monteiro, P.T., von Kamp, A., Xenarios, I., de Jong, H., Hucka, M., Klamt, S., Thieffry, D., Le Novère, N., Saez-Rodriguez, J., Helikar, T., 2013. SBML qualitative models: a model representation format and infrastructure to foster interactions between qualitative modelling formalisms and tools. BMC Syst. Biol. 7, 135. doi:10.1186/1752-0509-7-135

Chaouiya, C., Keating, S.M., Berenguier, D., Naldi, A., Thieffry, D., van lersel, M.P., Le Novère, N., Helikar, T., 2015. SBML Level 3 package : Qualitative Models , Version 1 , Release 1. J. Integr. Bioinform. 12. doi:10.2390/biecoll-jib-2015-270

Chaouiya, C., Naldi, A., Thieffry, D., 2012. Logical modelling of gene regulatory networks with GINsim. Methods Mol. Biol. 804, 463-79. doi:10.1007/978-1-61779-361-5_23

Helikar, T., Cutucache, C.E., Dahlquist, L.M., Herek, T.A., Larson, J.J., Rogers, J.A., 2015. Integrating interactive computational modeling in biology curricula. PLoS Comput. Biol. 11, e1004131. doi:10.1371/journal.pcbi.1004131

Helikar, T., Kochi, N., Konvalina, J., Rogers, J.A., 2010. Boolean modeling of biochemical networks. Open Bioinforma. J. 4. 
Helikar, T., Kowal, B., Madrahimov, A., Shrestha, M., Pedersen, J., Limbu, K., Thapa, I., Rowley, T., Satalkar, R., Kochi, N., Konvalina, J., Rogers, J.A., 2012. Bio-logic builder: a non-technical tool for building dynamical, qualitative models. PLoS One 7, e46417. doi:10.1371/journal.pone.0046417

Helikar, T., Kowal, B., McClenathan, S., Bruckner, M., Rowley, T., Wicks, B., Shrestha, M., Limbu, K., Rogers, J.A., 2012. The Cell Collective: Toward an open and collaborative approach to systems biology. BMC Syst. Biol. 6, 96. doi:10.1186/1752-0509-6-96

Helikar, T., Kowal, B., Rogers, J.A., 2013. A cell simulator platform: the cell collective. Clin. Pharmacol. Ther. 93, 393-5. doi:10.1038/clpt.2013.41

Helikar, T., Rogers, J.A., 2009. ChemChains: a platform for simulation and analysis of biochemical networks aimed to laboratory scientists. BMC Syst Biol 3, 58.

Müssel, C., Hopfensitz, M., Kestler, H.A., 2010. BoolNet--an R package for generation, reconstruction and analysis of Boolean networks. Bioinformatics 26, 1378-80. doi:10.1093/bioinformatics/btq124

Slepchenko, B.M., Schaff, J.C., Macara, I., Loew, L.M., 2003. Quantitative cell biology with the Virtual Cell. Trends Cell Biol. 13, 570-6.

Terfve, C.D.A., Cokelaer, T., Henriques, D., Macnamara, A., Gonçalves, E., Morris, M.K., van lersel, M., Lauffenburger, D.A., Saez-Rodriguez, J., 2012. CellNOptR: a flexible toolkit to train protein signaling networks to data using multiple logic formalisms. BMC Syst. Biol. 6, 133. doi:10.1186/1752-0509-6-133 